\title{
The Oosterhoff types of the Fornax dSph Globular Clusters
}

\author{
C. Greco ${ }^{1,2}$ G. Clementini ${ }^{1}$, M. Catelan ${ }^{3}$, E. Poretti ${ }^{4}$, E. V. Held ${ }^{5}$,

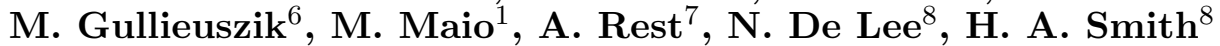 \\ and B. J. Pritzl ${ }^{9}$ \\ ${ }^{1}$ INAF- O.A.Bologna, Via Ranzani, 1, 40127, Bologna, Italy \\ email: claudia.greco@oabo.inaf.it \\ ${ }^{2}$ Astronomy Department, Bologna University, Via Ranzani, 1, 40127, Bologna, Italy \\ ${ }^{3}$ Departamento de Astronomía y Astrofísica, Pontificia Universidad Católica de Chile, Avenida \\ Vicuña Mackenna 4860, 782-0436 Macul, Santiago, Chile \\ ${ }^{4}$ INAF- O.A.Brera, Via E. Bianchi 46, 23807 Merate, Italy \\ ${ }^{5}$ INAF- O.A.Padova, Vicolo dell'Osservatorio 5, 35122, Padova, Italy \\ ${ }^{6}$ Astronomy Department, Padua University, vicolo dell'Osservatorio 2, 351222, Padova, Italy \\ ${ }^{7}$ Cerro Tololo Inter-American Observatory, Casilla 603, La Serena, Chile \\ ${ }^{8}$ Department of Physics and Astronomy, Michigan State University, East Lansing, MI \\ 48824-2320, USA \\ ${ }^{9}$ Macalester College, 1600 Grand Avenue, Saint Paul, MN 55105, USA
}

\begin{abstract}
We have applied the Image Subtraction to B,V time-series photometry of four globular clusters of the Fornax dwarf spheoridal galaxy, which were observed with the 2.2m ESO-MPI, the $6.5 \mathrm{~m}$ Magellan/Clay, the $4 \mathrm{~m}$ CTIO/Blanco telescopes and the WFPC2 on board of HST. We have identified RR Lyrae stars and obtained well sampled light curves for the clusters' variables. From the periods and pulsation properties of their RR Lyrae stars Fornax globular clusters seem to be of Oosterhoff intermediate type.
\end{abstract}

Keywords. Galaxies: dwarf -galaxies: individual(Fornax)-stars: variable -technique: photometry -technique: Image Subtraction

\section{Fornax globular clusters system}

Pulsating variable stars such as RR Lyrae stars, Dwarf and Anomalous Cepheids are very interesting astronomical objects that can be used to derive important information on the stellar system they belong to. They are easily identified, even in high crowding conditions, thanks to light variation, and their periods are independent from reddening and distance. The RR Lyrae stars, belonging to the oldest population, eye-witnessed the early epochs of galaxy formation and thus hold a crucial role for identifying the 'building blocks' that contributed to formation of the Galactic halo. In merging scenarios, the halo of the Milky Way (MW) has been assembled, at least in part, through the accretion of protogalactic fragments partially resembling the present-day dwarf Spheroidal (dSph) companions of the MW. The MW variables should thus have properties that were directly inherited from their 'ancestors'. Variable stars in the MW globular clusters (GCs) are found to sharply divide into two distinct groups according to the mean period of their fundamental mode RR Lyrae stars (RRab).This phenomenon is called the Oosterhoff (Oo) dichotomy (Oosterhoff 1939). Oo type I GCs have $\left\langle P_{a b}\right\rangle=0.55 \mathrm{~d}$, while Oo type II GCs have $\left\langle P_{a b}\right\rangle=0.64 \mathrm{~d}$. In the Milky Way there are no clusters with $\left\langle P_{a b}\right\rangle$ intermediate 


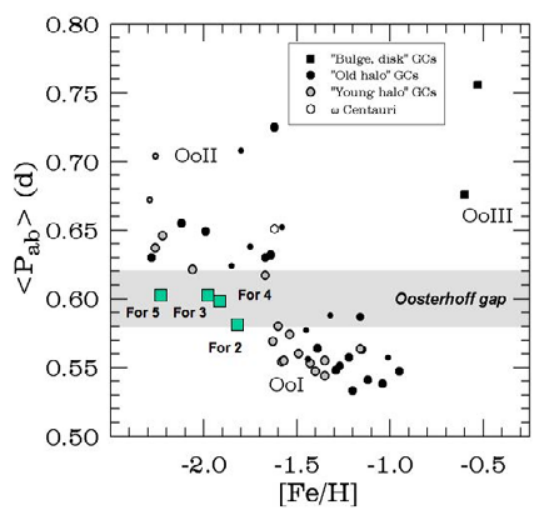

Figure 1. Positions on the Oosterhoff plane of different GC systems. Squares represent the Fornax dSph GCs: they seem to fill the gap defined by the Galactic GCs.

Table 1. RR Lyrae stars in the Fornax dSph GCs

\begin{tabular}{llllllcc}
\hline Cluster & {$[\mathrm{Fe} / \mathrm{H}]$} & RR Lyrae & $\left\langle\mathrm{P}_{a b}\right\rangle$ & $\left\langle\mathrm{P}_{c}\right\rangle$ & $\mathrm{B}$ & $\mathrm{V}$ & $\mathrm{I}$ \\
\hline For 2 & -1.79 & $32(13 \mathrm{ab}, 18 \mathrm{c}, 1 \mathrm{~d})$ & 0.578 & 0.356 & 6 & 32 & 16 \\
For 3 & -1.96 & $29(14 \mathrm{ab}, 5 \mathrm{c}, 10 \mathrm{~d})$ & 0.606 & 0.358 & 61 & 38 & 16 \\
For 4 & -1.90 & $29(22 \mathrm{ab}, 3 \mathrm{c}, 2 \mathrm{~d})$ & 0.599 & 0.358 & 80 & 201 & - \\
For 5 & -2.20 & $13(7 \mathrm{ab}, 5 \mathrm{c}, 1 \mathrm{~d})$ & 0.576 & 0.353 & 10 & 55 & 16 \\
\hline
\end{tabular}

between the two Oo types. The Fornax galaxy and Sagittarius are the only dSphs known to host GCs. Fornax GCs system contains five GCs. Do the Fornax GCs show an Oosterhoff dicothomy? If they do not, then they cannot be representatives of the protogalactic fragments from which the Galaxy's halo has been assembled.

Mackey \& Gilmore (2003) identified candidate RR Lyrae stars in Fornax 1, 2, 3 and 5 using HST archival data. They determined periods by fitting template RR Lyrae light curves to their data, and found that the Fornax clusters possess mean characteristic intermediate between the two Oo groups. Time-series B,V photometry of the Fornax clusters 2,3,4, and 5 was collected with the $2.2 \mathrm{~m}$ ESO-MPI and the $6.5 \mathrm{~m}$ Magellan/Clay telescopes, and combined with HST archival data and 4m CTIO/Blanco observations. Variable stars were identified with the Image Subtraction Technique (Alard, 2000). Photometry was performed with DAOPHOT-ALLSTAR-ALLFRAME (Stetson, 1994). We obtained well sampled light curves and derived periods for the variables in these 4 GCs (see Table 1). This is the first variability survey of For 4 ever performed (Greco et al. 2007).

The Fornax GCs are found to belong to an Oosterhoff-intermediate class and fill the Oosterhoff-gap defined by the MW GCs. Our findings allow us to exclude that the MW halo can have been assembled by stripping of Fornax dSph-like protogalactic fragments.

\section{References}

Alard, C. 2000, A\&AS 144, 363

Greco, C., Clementini, G., Catelan. M. et al. 2007, ApJ submitted

Mackey, A.D. \& Gilmore, G.F. 2003, MNRAS 345, 747

Oosterhoff, P.Th. 1939, Observatory 62, 104

Stetson, P.B. 1994, PASP 106, 250 